\title{
Structural Chemistry and Magnetic Properties of $\mathrm{Y}_{2} \mathrm{CoGe}_{4} \mathrm{O}_{12}$
}

\author{
Xiao-Qiang Liu ${ }^{1}$, Peter D. Battle ${ }^{2, *}$, Joe Ridout ${ }^{2}$, Diming $\mathrm{Xu}^{2}$ and \\ Silvia Ramos ${ }^{3, *}$
}

1. Department of Materials Science and Engineering, Zhejiang University, 38 Zheda Rd., Hangzhou 310027, China

2. Inorganic Chemistry Laboratory, Oxford University, South Parks Road, Oxford, OX1 3QR, U. K.

3. School of Physical Sciences, Ingram Building, University of Kent, Canterbury, Kent, CT2 7NH, U. K.

* Authors to whom correspondence should be addressed: peter.battle@chem.ox.ac.uk 


\begin{abstract}
Polycrystalline $\mathrm{Y}_{2} \mathrm{CoGe}_{4} \mathrm{O}_{12}$ has been prepared by standard ceramic methods. The crystal structure (space group P4/nbm; a = 9.8465(2), c = 4.92986(9) Å) consists of metal-rich layers separated from each other by $\mathrm{Ge}_{4} \mathrm{O}_{12}$ groups comprised of four corner-sharing $\mathrm{GeO}_{4}$ tetrahedra. Two cation sites lie within the layers; an eight-coordinate site occupied by yttrium and a six-coordinate site occupied by a 1:1 disordered distribution of yttrium and cobalt. Neutron diffraction revealed two-fold disorder on the oxide sublattice; this has been elucidated using Co K-edge EXAFS spectroscopy. The availability of two sites allows each oxide ion to accommodate the coordination preferences of its single $\mathrm{Co} / \mathrm{Y}$ neighbour; the $\mathrm{GeO}_{4}$ tetrahedra distort to absorb any consequent strain. The octahedron of anions around each $\mathrm{Co}^{2+}$ cation shows a pseudo-tetragonal distortion with a strain $(\mathrm{Co}-\mathrm{O})_{\mathrm{eq}}-(\mathrm{Co}-$ $\mathrm{O})_{\mathrm{ax}} /(\mathrm{Co}-\mathrm{O})_{\mathrm{eq}}=-0.173$. This results in an unusually large effective magnetic moment of $6.05 \mu_{\mathrm{B}}$ per $\mathrm{Co}^{2+}$ cation.
\end{abstract}

\title{
Introduction
}

Yamane et al [1] and Zubkov et al [2] have previously described the crystal structure of compositions in the family $L n_{2} M^{2+} \mathrm{Ge}_{4} \mathrm{O}_{12}$, where $L n$ is a lanthanide (a term that we shall take to include yttrium), and $M$ is $\mathrm{Ca}, \mathrm{Mn}$ or $\mathrm{Zn}$. The structure can be considered to consist of metal-rich layers separated from each other by $\mathrm{Ge}_{4} \mathrm{O}_{12}$ groups made up of four corner-sharing $\mathrm{GeO}_{4}$ tetrahedra. Two crystallographically distinct cation sites lie within the layers; an eightcoordinate site occupied exclusively by $L n$ and a six-coordinate site occupied by a disordered arrangement of $L n$ and $M$, see Figure 1. The structure adopts the tetragonal space group $P 4 / n b m$. Zubkov et al were motivated by an interest in the optical properties of $\mathrm{Ln}_{2} \mathrm{M}^{2+} \mathrm{Ge}_{4} \mathrm{O}_{12}$ [3-5]. They synthesized a number of different compositions and characterised them using X-ray powder diffraction. The temperature dependence of the magnetic 
susceptibility of $\mathrm{Eu}_{2} \mathrm{MnGe}_{4} \mathrm{O}_{12}$ was measured in an attempt to identify the oxidation states of the two metals, but no other magnetic data have been reported.

The occupation of a six-coordinate site by a disordered arrangement of $\mathrm{Ca}^{2+}$ and $\mathrm{Ln}^{3+}$ has been seen previously, for example in $\mathrm{Ca}_{2} \mathrm{HoRuO}_{6}$ [6], but the ionic radii of lanthanide cations and divalent cations of the first-row transition metals, for example $\mathrm{Mn}^{2+}$, are usually sufficiently different to exclude such mixing. We have therefore begun to explore which combinations of $\mathrm{Ln}$ and $M$ can be accommodated in this crystal structure. In contrast to Zubkov et al, our study was motivated by an interest in the magnetic properties of these compositions. In this paper we describe a detailed structural study of $\mathrm{Y}_{2} \mathrm{CoGe}_{4} \mathrm{O}_{12}$ that was undertaken following the observation of apparently anomalous magnetic behaviour. EXAFS was used to determine the local environments around the cobalt cations in order to facilitate a comparison with the average environment around the six-coordinate site as determined by neutron diffraction.

\section{Experimental}

Attempts were made to prepare polycrystalline samples of $L n_{2} M^{2+} \mathrm{Ge}_{4} \mathrm{O}_{12}(\mathrm{M}=\mathrm{Fe}$, Co) by heating stoichiometric mixtures of $\mathrm{GeO}_{2}$, dried $\mathrm{Ln}_{2} \mathrm{O}_{3}$ and either $\mathrm{Fe}_{3} \mathrm{O}_{4}$ or $\mathrm{Co}_{3} \mathrm{O}_{4}$ in air for $\sim 100$ hours, initially at $1050{ }^{\circ} \mathrm{C}$. All compositions apart from $\mathrm{Y}_{2} \mathrm{CoGe}_{4} \mathrm{O}_{12}$ were subsequently heated at $1075{ }^{\circ} \mathrm{C}$. In the light of the results of the magnetic study of $\mathrm{Y}_{2} \mathrm{CoGe}_{4} \mathrm{O}_{12}$ it was decided to study the magnetic behaviour of the magnetically-diluted compositions $\mathrm{Y}_{2} \mathrm{Co}_{1}$ ${ }_{y} \mathrm{Ca}_{y} \mathrm{Ge}_{4} \mathrm{O}_{12}(0<y<1) . \mathrm{CaCO}_{3}$ was used as a starting material in the syntheses of these compounds, which took $150 \mathrm{~h}$ to complete at a temperature of $1075{ }^{\circ} \mathrm{C}$; the reactants were reground every $20 \mathrm{~h}$. The progress of all the reactions, the purities of the products and, in the case of a successful reaction, the crystal structure of the product were studied using X-ray powder diffraction. Data collected at room temperature on a Panalytical X'Pert diffractometer 
operating with $\mathrm{Cu} \mathrm{K} \alpha_{1}$ radiation were analysed by the Rietveld method [7], as implemented in the FullProf program suite [8]. The magnetic susceptibility of selected compositions was measured over the temperature range $2<\mathrm{T} / \mathrm{K}<300$ using a Quantum Design MPMS 5000 SQUID magnetometer. Data were collected on warming in a field of 100 Oe after cooling the sample both in the absence of an applied field (zero-field cooled, ZFC) and in the measuring field (field cooled, FC).

On the basis of the results of these measurements one sample, $\mathrm{Y}_{2} \mathrm{CoGe}_{4} \mathrm{O}_{12}$, was selected for further study. The diffractometer D2b at ILL Grenoble was used to collect neutron powder diffraction data at a wavelength of $\sim 1.59 \AA$. The sample was contained in a vanadium can. Data were collected over the angular range $10<2 \theta /{ }^{\circ}<155$ with a step size $\Delta 2 \theta=0.05^{\circ}$. They were analysed by the Rietveld method as implemented in the GSAS program package [9]. Xray absorption spectra were collected on beamline B18 at the Diamond Light Source from the same $L n=$ Y sample. A well-ground sample was diluted with cellulose before being pressed into a pellet of $8 \mathrm{~mm}$ diameter under a pressure of 1.5 tonnes. Data were collected at the Co K-absorption edge in transmission using the QEXAFS mode (that is, data were collected as the monochromator scanned in energy continuously, without stopping other than at the limits of the required energy range). The beamline was set-up to use the Si(111) crystals in the monochromator and the Cr coating of the different mirrors. Data were collected between room temperature and $100 \mathrm{~K}$ using a liquid nitrogen cryostat. Here we will present only the results obtained at $300 \mathrm{~K}$ and $100 \mathrm{~K}$ as no structural transitions were observed in the intermediate temperature region. Data analysis was carried out using the programs Athena and Artemis [10].

\section{Results}


Syntheses involving iron were unsuccessful and will not be discussed further. Bragg peaks attributable to the phase described by Zubkov et al [2] could be seen in the X-ray diffraction patterns of $L n_{2} \mathrm{CoGe}_{4} \mathrm{O}_{12}$ for $L n=\mathrm{Y}, \mathrm{Gd}$, Tb, Ho, Er, Yb and Lu. However, for all lanthanides other than yttrium the samples contained a significant $(2.9-6.4 \%)$ level of $L n_{2} \mathrm{Ge}_{2} \mathrm{O}_{7}$ impurity. In the case of $\mathrm{Y}_{2} \mathrm{CoGe}_{4} \mathrm{O}_{12}, \mathrm{X}$-ray diffraction showed the reaction product to consist largely of a phase isostructural with those prepared by Zubkov et al, with only $\sim 1$ weight per cent $\mathrm{Y}_{2} \mathrm{Ge}_{2} \mathrm{O}_{7}$ present. No impurity was detected when the ratio of the reactants was modified to give a nominal composition of $\mathrm{Y}_{1.95} \mathrm{CoGe}_{4} \mathrm{O}_{12}$ and this sample was used in our subsequent studies. The unit cell parameters refined to $a=9.8465(2), c=$ 4.92986(9) Å. The temperature dependence of the molar magnetic susceptibility of this sample is shown in Figure 2. No phase transition is apparent in the measured temperature range. Fitting the data in the range $150<\mathrm{T} / \mathrm{K}<300$ to the Curie-Weiss law resulted in a Weiss temperature $\theta=-27.4(4) \mathrm{K}$ and a Curie constant $\mathrm{C}=4.581(9) \mathrm{cm}^{3} \mathrm{~K} \mathrm{~mol}^{-1}$. The latter leads to a value of $6.05 \mu_{\mathrm{B}}$ for the effective magnetic moment of the cobalt cations. This result was reproduced in measurements made on independently-synthesized samples.

The neutron diffraction pattern of the yttrium-containing sample could be accounted for in the space group $P 4 / n b m$; no reflections attributable to an impurity phase were detected in a visual inspection of the data. In the analysis of these data the unit cell parameters were held constant at the values determined from the X-ray data and the wavelength of the neutron beam was refined. Initially single-phase refinements were carried out using the structural model described by Zubkov et al, with a disordered distribution of yttrium and cobalt on the $4 f$ site. The yttrium content of the $2 b$ and $4 f$ sites was refined but no significant deficiency was detected on either site. During the course of the data analysis it was noted that the displacement parameter of the oxide ion on the $16 i$ site in Zubkov's model was relatively large. This atom was therefore disordered over two half-occupied 16i sites, O2 and O3, which 
were constrained to have the same displacement parameter. Despite the absence of clearly visible reflections from a second phase, in order to allow for the yttrium-deficient composition of the reaction mixture, the phase $\mathrm{CoGeO}_{3}$ [11] was included in our final refinements. The presence of this weakly-scattering phase was anticipated because it has been formed in relatively large amounts during our attempts to synthesize related compounds under similar conditions. Our refinements showed that in this case it constituted 0.6(1) weight per cent, that is 2.5 mole per cent, of the reaction product. The final observed and calculated diffraction patterns are shown in Figure 3; the agreement factors are $\mathrm{R}_{\mathrm{wpr}}=5.0 \%, \chi^{2}=4.9$. The resulting structural parameters are listed in Table 1 and the corresponding bond lengths and angles are presented in Table 2 . The atom $\mathrm{Y} 1$ on the $2 b$ site is surrounded by a total of eight O2/O3 anions, distributed in a disordered manner over the half-occupied $16 n$ sites. The difference between the $\mathrm{Y} 1$ - $\mathrm{O} 2$ and $\mathrm{Y} 1$ - O3 distances is not significant and the oxide ions form a square antiprism around the cation. The $4 f$ site, where Co and Y2 are accommodated, formally has $2 / \mathrm{m}$ point symmetry but can be considered to be coordinated by a tetragonallydistorted octahedron of oxide ions; there are two long bonds to O1 and four shorter bonds to either $\mathrm{O} 2$ or $\mathrm{O} 3$. The $\mathrm{GeO}_{4}$ tetrahedra contain two $\mathrm{Ge}-\mathrm{O} 1$ bonds and two $\mathrm{Ge}-\mathrm{O} 2 / \mathrm{O} 3$ bonds. The former are longer than the latter; there is no significant difference between the Ge - O2 and Ge - O3 distances. The partially occupied O2 and O3 sites are 0.153(10) $\AA$ apart; the shortest real $\mathrm{O}-\mathrm{O}$ distance in the structure is the $\mathrm{O} 1-\mathrm{O} 2$ contact at 2.743(5) $\AA$.

The X-ray absorption data show no changes in either the atomic structure or the oxidation state of Co in the sample between $300 \mathrm{~K}$ and $100 \mathrm{~K}$, see Figure 4. The fitted EXAFS data for the local structure around the $\mathrm{Co}^{2+}$ cation at the two temperatures are shown in Figure 5. The data sets for the two temperatures were fitted simultaneously using a single model. The results from the fit are described in detail in Table 3. The first peak can be modelled using the symmetry defined by the diffraction results, that is the six oxide ions surrounding a $\mathrm{Co}^{2+}$ ion 
are split into a group of four with a Co-O distance, R, of 2.064(1) $\AA$ at $100 \mathrm{~K}$ (2.072(9) $\AA$ at $300 \mathrm{~K}$ ) and a group of two further away at 2.43(5) $\AA$. The higher standard deviation on the radial distance and the larger value of the Debye-Waller factor, $\sigma$, show that the two oxide ions at the longer distance from the cobalt are significantly more disordered that the closer group. The level of disorder is such that these anions, $\mathrm{O} 1$ in Table 1, do not make a detectable contribution to the spectra beyond the first peak. The second peak is broad and, according to the theoretical model, should encompass a large number of single and multiple scattering paths. Different fitting strategies that included all the main single-scattering paths in this region were attempted and good fits were achieved. However it was found that the resulting values of the interatomic distance and Debye-Waller factor for the Co-Y path were unphysical. Consequently, a new strategy was followed in which the data were modelled without including the Co-Y path, and this resulted in a comparably good fit. As this second approach made use of a smaller number of parameters for the fit (19 out of 32 independent points as opposed to 22), it was concluded that this was a better model.

We were able to prepare samples of $\mathrm{Y}_{2} \mathrm{Co}_{1-y} \mathrm{Ca}_{y} \mathrm{Ge}_{4} \mathrm{O}_{12}$ for $y=0.25,0.5$; attempts to prepare samples with $0.5<y<1.0$ did not result in monophasic reaction products. The parameters derived from our X-ray diffraction and magnetometry studies of these two compositions are presented in Table 4. The X-ray data were analysed using Zubkov's model with an ordered oxide sublattice. The bond lengths labelled Co - O1 and Co - O2 in Table 4 are the mean values of those around the $4 f$ site that accommodates a disordered arrangement of cobalt, calcium and yttrium. The temperature dependence of the molar magnetic susceptibility is shown in Figure S1.

\section{Discussion}


Our attempts to prepare $L n_{2} M^{2+} \mathrm{Ge}_{4} \mathrm{O}_{12}(\mathrm{M}=\mathrm{Fe}, \mathrm{Co})$ met with limited success compared to the manganese-containing syntheses performed by Zubkov et al. This presumably stems from a decrease in the relative thermodynamic stability of $L n_{2} M G e_{4} \mathrm{O}_{12}$ when the ionic radius of $M^{2+}$ decreases below that of $\mathrm{Mn}^{2+}$. The purity of the product in the yttrium-cobalt system apparently improved when the reaction mixture was made yttrium deficient although the composition of the main phase, as determined from the neutron diffraction data, did not differ significantly from the original target composition, $\mathrm{Y}_{2} \mathrm{CoGe}_{4} \mathrm{O}_{12}$. The need to use an yttriumdeficient reaction mixture is explained by the presence of 2.5 mole per cent of a $\mathrm{CoGeO}_{3}$ impurity. Other impurities, which must be present in order to balance the stoichiometry of the reaction mixture, were not present in high enough concentrations to enable them to be identified. Our spectroscopic data confirm the oxidation state of the cobalt in our sample to be $\mathrm{Co}^{2+}$; this is consistent with the presence of both $\mathrm{Y}_{2} \mathrm{CoGe}_{4} \mathrm{O}_{12}$ and a $\mathrm{CoGeO}_{3}$ impurity. The Co - O bond length in cubic $\mathrm{CoO}$ is $2.129 \AA$ [12] and the mean $\mathrm{Y}-\mathrm{O}$ distance $\operatorname{in}_{2} \mathrm{O}_{3}$ is $2.272 \AA$ [13]. Taking the radius of the oxide ion to be $1.4 \AA$, these bond lengths are largely consistent with the tabulated six-coordinate ionic radii of $\mathrm{Co}^{2+}$ and $\mathrm{Y}^{3+}, 0.745$ and $0.900 \AA$ respectively [14]. A difference in radii of $\sim 0.15 \AA$ might be expected to result in the ordering of two cation species over different crystallographic sites, but our neutron diffraction data show that this is not the case in $\mathrm{Y}_{2} \mathrm{CoGe}_{4} \mathrm{O}_{12}$; the transition metal and the lanthanide occupy the $4 f$ site in a 1:1 disordered array. If we assume for the present that each $4 f$ cation is bonded to $2 \times \mathrm{O} 1,2 \times \mathrm{O} 2$ and $2 \times \mathrm{O}$, i.e. a truly disordered arrangement, the mean bond length at the 4f site is $2.230 \AA$, which is short for $\mathrm{Y}^{3+}$ and long for $\mathrm{Co}^{2+}$. When analysed in terms of the bond-valence sum (BVS) [15] the $\mathrm{Y}^{3+}$ and $\mathrm{Co}^{2+}$ cations at these sites appear to be overbonded (BVS = 3.59) and under-bonded (BVS = 1.50), respectively. These BVS values confirm that the mean bond lengths are unusually small and large for six-coordinate $\mathrm{Y}^{3+}$ and $\mathrm{Co}^{2+}$, respectively. 
There are thus two noteworthy features in the description of the structure of $\mathrm{Y}_{2} \mathrm{CoGe}_{4} \mathrm{O}_{12}$ derived from the neutron diffraction data. It is unusual for a transition metal and a lanthanide to occupy the same site in a 1:1 disordered arrangement, and the average bond length around the six-coordinate site in $\mathrm{Y}_{2} \mathrm{CoGe}_{4} \mathrm{O}_{12}$ is inconsistent with the tabulated ionic radii of both $\mathrm{Co}^{2+}$ and $\mathrm{Y}^{3+}$. This suggests that the local environments around the different types of cation might differ significantly from the average structure determined by neutron diffraction. The EXAFS experiments described above were performed in an attempt to understand these anomalies by determining the local structure around the $\mathrm{Co}^{2+}$ cations at the $4 f$ sites. Comparison of the data presented in Table 3 with those in Table 2 shows firstly that the local environment around the $\mathrm{Co}^{2+}$ cations does indeed differ significantly from the average environment determined by neutron diffraction. The mean Co - O distance determined by EXAFS is $2.19 \AA$ at $300 \mathrm{~K}$, and the BVS around this cation is 1.70 . These values are closer to the expected values than those derived from the diffraction data but they are still inconsistent with an ionic description of the bonding. The EXAFS data have allowed us to determine directly the bond lengths in the coordination octahedron around the $4 f$ site when it is occupied by cobalt. The four-fold equatorial bond length determined by EXAFS is shorter than both the $\mathrm{Co}-\mathrm{O} 2$ and $\mathrm{Co}-\mathrm{O} 3$ bond lengths determined by diffraction, see Tables 2 and 3, but closer to the former. We conclude that, in the model used to analyse the diffraction data, the $\mathrm{O} 2$ and $\mathrm{O} 3$ anions are not truly disordered but that the $\mathrm{O} 2$ and $\mathrm{O} 3$ sites model the anion position when the $4 f$ site is occupied by cobalt and yttrium, respectively; that is, each $\mathrm{Co}^{2+}$ cation is coordinated by 2 x $\mathrm{O} 1$ and 4 x O2, whereas each $\mathrm{Y}^{3+}$ is coordinated by $2 \times \mathrm{O} 1$ and 4 x O3. The key feature of this structure that allows occupation of one crystallographic site by two cations having significantly different sizes is that no oxide ion is coordinated to more than one $4 f$ site, and hence the choice of anion site is never frustrated. Thus, although the $\mathrm{Y}^{3+}$ and $\mathrm{Co}^{2+}$ cations are randomly distributed over the $4 f$ sites, the anions are able to respond to 
the coordination requirements of their nearest cation. No disorder on the axial $8 m$ anion site accommodating $\mathrm{O} 1$ could be detected in our neutron data although the EXAFS data suggest that some is present. The difference of 0.018(8) $\AA$ between the Y1 - O2 and Y1 - O3 distances determined by neutron diffraction is small but it is likely that the location of the Y1 site shows some disorder depending on the local distribution of the eight neighbouring O2/O3 anions. This might explain why the Y1 cation has little impact on the Co EXAFS data. As we remarked above, the disorder on the $16 n$ anion site also has little impact on the bond lengths to the $8 k$ Ge site. No anomalously short $\mathrm{O}-\mathrm{O}$ contacts are introduced, although the $\mathrm{O}-\mathrm{Ge}-$ O bond angles deviate from their ideal value, see Table 2. The extent of this deviation is comparable to, but slightly larger than, that reported for related compounds [1]. It is possible that the range of cation pairs that can occupy the $4 f$ site is ultimately determined by the limited flexibility of the $\mathrm{GeO}_{4}$ tetrahedra. The $\mathrm{Co}-\mathrm{O}$ distance in the second coordination shell as determined by EXAFS, 3.56(4) $\AA$, is consistent with the Co - O2 and Co - O3 distances of 3.498(6) and 3.597(6) $\AA$, respectively, determined by neutron diffraction. However, the third-nearest-neighbour Co - O distances derived from the neutron diffraction data are 4.167(5) and 4.079(5) $\AA$ for $\mathrm{Co}$ - O2 and Co - O3 whereas the distance derived from the EXAFS data is 4.03(2) Å. This mismatch might reflect the limitations of the way in which we have modelled the oxygen disorder around the $4 f$ site when analysing our diffraction.

The detailed structural study described above was prompted by the observation that the effective magnetic moment, $6.05 \mu_{\mathrm{B}}$, of the $\mathrm{Co}^{2+}$ cations in $\mathrm{Y}_{2} \mathrm{CoGe}_{4} \mathrm{O}_{12}$ is unusually large. The absence of any magnetic phase transition above $2 \mathrm{~K}$ in this composition suggests that the anomaly is not a consequence of intercation interactions but is a property of the individual $\mathrm{Co}^{2+}$ cations. This conclusion is supported by the observation of similarly large moments in the magnetically-diluted compositions $\mathrm{Y}_{2} \mathrm{Co}_{1-y} \mathrm{Ca}_{y} \mathrm{Ge}_{4} \mathrm{O}_{12}$. The value of the effective magnetic moment of six-coordinate, high-spin $\mathrm{Co}^{2+}$ calculated using the spin-only formula is 
$3.87 \mu_{\mathrm{B}}$. Experimentally-determined values usually lie in the range $4.7-5.3 \mu_{\mathrm{B}}[16,17]$. This level of enhancement above the spin-only value is attributable to an orbital contribution to the moment that stems from the residual orbital degeneracy of the ${ }^{4} \mathrm{~T}_{1 \mathrm{~g}}$ ground state in regular octahedral coordination. The value observed in a particular compound depends on the relative magnitude of the spin-orbit coupling constant and the non-cubic component of the crystal field around the cation [18, 19]. Our neutron diffraction and EXAFS data show that the octahedra around the $4 f$ sites are tetragonally distorted with $c / a>1$. In this case the orbital contribution to the moment is initially reduced as the ${ }^{4} \mathrm{~T}_{1 \mathrm{~g}}$ state splits into a ${ }^{4} \mathrm{E}_{\mathrm{g}}$ state and a ${ }^{4} \mathrm{~A}_{2 \mathrm{~g}}$ state, with the latter, which can be represented by the electron configuration $\left(d_{\mathrm{xz}}, d_{\mathrm{yz}}\right)^{4}$ $d_{\mathrm{xy}}{ }^{1} d_{\mathrm{z} 2}{ }^{1} d_{\mathrm{x} 2-\mathrm{y} 2}{ }^{1}$, being lower in energy. However, as discussed by Smura et al [20], when the tetragonal distortion is large the $3 d_{\mathrm{z} 2}$ orbital is lowered in energy sufficiently for it to become accessible. In the limiting case of square-planar geometry it can become the lowest-energy member of the $d$ manifold and the electron configuration of $\mathrm{Co}^{2+}$ can then be written as $d_{\mathrm{zz}}{ }^{2}$ $\left(d_{\mathrm{xz}}, d_{\mathrm{yz}}\right)^{3} d_{\mathrm{xy}}{ }^{1} d_{\mathrm{x} 2-\mathrm{y} 2}{ }^{1}$. The availability of the $3 d_{\mathrm{z} 2}$ orbital restores orbital degeneracy to the ground state and the magnetic moment increases again. We therefore propose that the unusually high effective moment observed in $\mathrm{Y}_{2} \mathrm{CoGe}_{4} \mathrm{O}_{12}$ is a consequence of the large tetragonal distortion of the $\mathrm{CoO}_{6}$ octahedra therein. The magnetic properties of $\mathrm{Y}_{2} \mathrm{Co}_{1}$ ${ }_{y} \mathrm{Ca}_{y} \mathrm{Ge}_{4} \mathrm{O}_{12}$ are consistent with this explanation, see Table 4. The magnitude of the strain, $((\mathrm{Co}-\mathrm{O} 2)-(\mathrm{Co}-\mathrm{O} 1)) /(\mathrm{Co}-\mathrm{O} 2)=-0.173$, in $\mathrm{Y}_{2} \mathrm{CoGe}_{4} \mathrm{O}_{12}$ can be compared to the much smaller value found [21] in the low-temperature, pseudo-tetragonal form of $\mathrm{CoO}$ where ((Co $-\mathrm{O} 2)-(\mathrm{Co}-\mathrm{O} 1)) /(\mathrm{Co}-\mathrm{O} 2)=0.012$.

\section{Conclusions}

This work began as a wide-ranging study of the system $\mathrm{Ln}_{2} \mathrm{M}^{2+} \mathrm{Ge}_{4} \mathrm{O}_{12}$ but became focussed on one particular composition, $\mathrm{Y}_{2} \mathrm{CoGe}_{4} \mathrm{O}_{12}$. Neutron powder diffraction confirmed that 
cobalt and yttrium are distributed randomly in a 1:1 ratio over a single crystallographic site in the tetragonal crystal structure and also revealed the presence of disorder on the oxide sublattice around the Co/Y sites. EXAFS spectroscopy was used to study specifically the local coordination environment of the $\mathrm{Co}^{2+}$ cations and to determine the $\mathrm{Co}-\mathrm{O}$ bondlengths therein. These data have then allowed us to interpret the anion disorder revealed by the diffraction experiment. The large tetragonal strain in the $\mathrm{CoO}_{6}$ octahedra is consistent with the observation of a large effective magnetic moment for the $\mathrm{Co}^{2+}$ cations. This study demonstrates the benefits of using a combination of diffraction and spectroscopic techniques in the study of a structurally-complex, disordered material.

\section{Acknowledgements}

We thank E. Suard for experimental assistance at ILL Grenoble and we thank STFC for the provision of beamtime at the Diamond Light Source.

\section{References}

1. H. Yamane, R. Tanimura, T. Yamada, J. Takahashi, T. Kajiwara and M. Shimada, J. Solid State Chem. 179, 289-295 (2006)

2. V. G. Zubkov, N. V. Tarakina, Leonidov, II, A. P. Tyutyunnik, L. L. Surat, M. A. Melkozerova, E. V. Zabolotskaya and D. G. Kellerman, Journal of Solid State Chemistry 183, 1186-1193 (2010)

3. I. I. Leonidov, V. G. Zubkov, A. P. Tyutyunnik, N. V. Tarakina, L. L. Surat, O. V. Koryakova and E. G. Vovkotrub, J. Alloys and Compounds 509, 1339 (2011)

4. M. A. Melkozerova, N. V. Tarakina, L. G. Maksimova, A. P. Tyutyunnik, L. L. Surat, I. I. Leonidov, V. G. Zubkov, E. V. Zabolotskaya and R. F. Samigullina, J. Sol-Gel Sci. Technol. 59, 338 (2011)

5. I. I. Leonidov, V. P. Petrov, V. A. Chernyshev, A. E. Nikiforov, E. G. Vovkotrub, A. P. Tyutyunnik and V. G. Zubkov, J. Phys. Chem. C 118, 8090 (2014)

6. P. D. Battle, C. W. Jones and F. Studer, J. Solid State Chem. 90, 302 (1991)

7. $\quad$ H. M. Rietveld, Journal of Applied Crystallography 2, 65 (1969) 
8. J. Rodriguez-Carvajal, Physica B 192, 55 (1993)

9. A. C. Larson and R. B. von Dreele, General Structural Analysis System (GSAS), Los Alamos National Laboratories, Report LAUR 86-748, 1990

10. B. Ravel and M. Newville, J. Synchrotron Radiation 12, 537 (2005)

11. G. J. Redhammer, A. Senyshyn, G. Tippelt, C. Pietzonka, G. Roth and G. Amthauer, Phys. Chem. Minerals 37, 311 - 332 (2010)

12. N. C. Tombs and H. P. Rooksby, Nature 165, 442 (1950)

13. M. G. Paton and E. N. Maslen, Acta Crystallographica 19, 307 (1965)

14. R. D. Shannon, Acta Crystallographica A 32, 519 (1976)

15. N. E. Brese and M. O'Keeffe, Acta Crystallographica B 47, 192 (1991)

16. E. K. Barefield and D. H. Busch, Q. Rev. Chem. Soc. 22, 457 (1968)

17. J. R. Singer, Phys . Rev. 104, 929 (1956)

18. J. B. Goodenough, Physical Review 171, 466 (1968)

19. A. Abragam and M. H. L. Pryce, Proceedings of the Royal Society of London Series A - Mathematical and Physical Sciences 206, 173-191 (1951)

20. C. F. Smura, D. R. Parker, M. Zbiri, M. R. Johnson and Z. A. Gal, J. Amer. Chem. Soc. 133, 2691 (2011)

21. W. Jauch, M. Reehuis, H. J. Bleif and F. Kubanek, Phys. Rev. B 64, 052102 (2001) 
Table 1 Structural parameters of $\mathrm{Y}_{2} \mathrm{CoGe}_{4} \mathrm{O}_{12}$ at room temperature.

\begin{tabular}{lllllll}
\hline Atom & Site & $x$ & $y$ & $z$ & $U_{\text {iso }} / \AA^{2}$ & Occupancy \\
\hline Y1 & $2 b$ & $1 / 4$ & $1 / 4$ & $1 / 2$ & $0.0059(6)$ & 1 \\
Y2 & $4 f$ & 0 & 0 & $1 / 2$ & $0.0070(8)$ & 0.5 \\
Co & $4 f$ & 0 & 0 & $1 / 2$ & $0.0070(8)$ & 0.5 \\
Ge & $8 k$ & $0.5220(1)$ & $1 / 4$ & 0 & $0.0043(2)$ & 1 \\
O1 & $8 m$ & $0.1299(1)$ & $0.8701(1)$ & $0.8315(3)$ & $0.0063(3)$ & 1 \\
O2 & $16 n$ & $0.1694(6)$ & $0.0571(6)$ & $0.2662(10)$ & $0.0049(4)$ & 0.5 \\
O3 & $16 n$ & $0.0683(6)$ & $0.1642(6)$ & $0.7527(10)$ & $0.0049(4)$ & 0.5
\end{tabular}

Space group $P 4 / n b m$ ( $2^{\text {nd }}$ setting); $a=9.8465, c=4.92986 \AA$

Table 2 Bond lengths $(\AA)$ and bond angles (degrees) in $\mathrm{Y}_{2} \mathrm{CoGe}_{4} \mathrm{O}_{12}$ at room temperature.

\begin{tabular}{|c|c|c|c|}
\hline $\mathrm{Y} 1-\mathrm{O} 2 *$ x 8 & $2.357(6)$ & $\mathrm{O} 1-\mathrm{Y} 1 / \mathrm{Co}-\mathrm{O} 1$ & 180 \\
\hline \multirow{2}{*}{$\mathrm{Y} 1-\mathrm{O}^{*}$ x 8} & $2.339(6)$ & $\mathrm{O} 1-\mathrm{Y} 1 / \mathrm{Co}-\mathrm{O} 2 *$ & 95.23 \\
\hline & & $\mathrm{O} 1-\mathrm{Y} 1 / \mathrm{Co}-\mathrm{O} 3 *$ & 80.90 \\
\hline $\mathrm{Y} 2 / \mathrm{Co}-\mathrm{O} 1$ x 2 & $2.438(2)$ & & \\
\hline $\mathrm{Y} 2 / \mathrm{Co}-\mathrm{O} 2 *$ x 4 & $2.107(6)$ & $\mathrm{O} 1-\mathrm{Ge}-\mathrm{O} 1$ & $107.4(1)$ \\
\hline \multirow{2}{*}{$\mathrm{Y} 2 / \mathrm{Co}-\mathrm{O}^{*}$ x 4} & $2.146(6)$ & $\mathrm{O} 1-\mathrm{Ge}-\mathrm{O} 2 *$ & $108.6(2)$ \\
\hline & & $\mathrm{O} 1-\mathrm{Ge}-\mathrm{O}^{*}$ & $107.5(2)$ \\
\hline $\mathrm{Ge}-\mathrm{O} 1 \times 2$ & $1.7933(9)$ & $\mathrm{O} 2 *-\mathrm{Ge}-\mathrm{O} 2 *$ & $126.0(4)$ \\
\hline $\mathrm{Ge}-\mathrm{O} 2 *$ x 2 & $1.719(5)$ & $\mathrm{O} 3^{*}-\mathrm{Ge}-\mathrm{O} 3^{*}$ & $118.2(4)$ \\
\hline $\mathrm{Ge}-\mathrm{O}^{*} \times 2$ & $1.732(5)$ & $\mathrm{O} 2 *-\mathrm{Ge}-\mathrm{O} 3 *$ & $122.0(1)$ \\
\hline $\mathrm{O} 2 *-\mathrm{O}^{*}$ & $0.153(10)$ & & \\
\hline
\end{tabular}

* indicates a site with $50 \%$ occupancy 
Table 3 Parameters obtained from refinements against Co K-edge EXAFS measurements for $\mathrm{Y}_{2} \mathrm{CoGe}_{4} \mathrm{O}_{12}$ at $300 \mathrm{~K}$ and $100 \mathrm{~K}$.

\begin{tabular}{|c|c|c|c|c|}
\hline & & & $300 \mathrm{~K}$ & $100 \mathrm{~K}$ \\
\hline $\mathrm{S}_{0}{ }^{2}$ & & & \multicolumn{2}{|c|}{$0.82(8)$} \\
\hline $\mathrm{E}_{0} / \mathrm{eV}$ & & & \multicolumn{2}{|c|}{$2.1(8)$} \\
\hline $\mathrm{R}$ Co $-\mathrm{O} 1 / \AA$ & \multirow{2}{*}{\multicolumn{2}{|c|}{$(\mathrm{N}=2)$}} & $2.46(4)$ & $2.43(5)$ \\
\hline$\sigma^{2}$ Co $-\mathrm{O} 1 / \AA^{2}$ & & & $0.014(7)$ & $0.010(9)$ \\
\hline $\mathrm{R} \mathrm{Co}-\mathrm{O} 2 / \AA$ & \multicolumn{2}{|l|}{$(\mathrm{N}=4)$} & $2.072(8)$ & $2.06(1)$ \\
\hline$\sigma^{2} \mathrm{Co}-\mathrm{O} 2 / \AA^{2}$ & & & $0.004(1)$ & $0.003(2)$ \\
\hline $\mathrm{R}$ Co - Ge / & \multicolumn{2}{|l|}{$(\mathrm{N}=8)$} & $3.46(1)$ & $3.46(1)$ \\
\hline$\sigma^{2} \mathrm{Co}-\mathrm{Ge} / \AA^{2}$ & & & $0.009(1)$ & $0.006(1)$ \\
\hline $\mathrm{R} \mathrm{Co}-\mathrm{O} 2 / \AA$ & \multicolumn{2}{|l|}{$(\mathrm{N}=4)$} & $3.56(4)$ & $3.54(5)$ \\
\hline$\sigma^{2} \mathrm{Co}-\mathrm{O} 2 / \AA^{2}$ & & & $0.005(3)$ & $0.003(3)^{*}$ \\
\hline $\mathrm{R} \mathrm{Co}-\mathrm{O} 2 / \AA$ & \multicolumn{2}{|l|}{$(\mathrm{N}=8)$} & $4.03(2)$ & $4.04(3)$ \\
\hline \multicolumn{3}{|l|}{$\sigma^{2} \mathrm{Co}-\mathrm{O} 2 / \AA^{2}$} & $0.005(3)^{*}$ & $0.003(3)^{*}$ \\
\hline $\mathrm{R} \mathrm{Co}-\mathrm{O} 2-\mathrm{O} 2 / \AA$ & $(\mathrm{N}=4)$ & (non-linear) & $4.14(1)^{*}$ & $4.13(1)^{*}$ \\
\hline \multicolumn{2}{|l|}{$\sigma^{2} \mathrm{Co}-\mathrm{O} 2-\mathrm{O} 2 / \AA^{2}$} & (non-linear) & $0.008(1)^{*}$ & $0.007(2)^{*}$ \\
\hline \multicolumn{2}{|c|}{$\mathrm{R} \mathrm{Co}-\mathrm{O} 2-\mathrm{O} 2 / \AA \quad(\mathrm{N}=4)$} & (linear, through absorber) & $4.14(1)^{*}$ & $4.13^{*}$ \\
\hline \multicolumn{2}{|l|}{$\sigma^{2} \mathrm{Co}-\mathrm{O} 2-\mathrm{O} 2 / \AA^{2}$} & (linear, through absorber) & $0.008(1)^{*}$ & $0.007(2)^{*}$ \\
\hline \multicolumn{3}{|c|}{ Number of independent points } & \multicolumn{2}{|c|}{32} \\
\hline \multicolumn{3}{|c|}{ Number of independent parameters } & \multicolumn{2}{|c|}{19} \\
\hline \multicolumn{3}{|l|}{ R-factor } & \multicolumn{2}{|c|}{0.023} \\
\hline
\end{tabular}

* These parameters were not allowed to vary freely in the fit, instead they have been linked to other parameters assumed to be either identical for geometrical reasons or expected to be very similar. 
Table 4 Structural and magnetic parameters of $\mathrm{Y}_{2} \mathrm{Co}_{1-y} \mathrm{Ca}_{y} \mathrm{Ge}_{4} \mathrm{O}_{12}$.

\begin{tabular}{lll}
\hline & $y=0.25$ & $y=0.50$ \\
\hline$a / \AA$ & $9.8807(1)$ & $9.9196(1)$ \\
$c / \AA$ & $4.96425(7)$ & $4.9992(1)$ \\
$\mu_{e f f} / \mu_{\mathrm{B}}^{*}$ & $6.01(4)$ & $5.99(9)$ \\
$\theta / \mathrm{K}^{*}$ & $-18.5(5)$ & $-18.4(10)$ \\
$\mathrm{Co}-\mathrm{O} 1 \times 2$ & $2.479(8)$ & $2.481(8)$ \\
$\mathrm{Co}-\mathrm{O} 2 \times 4$ & $2.154(6)$ & $2.170(6)$ \\
\hline
\end{tabular}

* derived by fitting $\chi^{-1}(\mathrm{~T})$ to the Curie-Weiss law 


\section{Figure Captions}

Figure 1 The crystal structure of $\mathrm{Ln}_{2} \mathrm{MGe}_{4} \mathrm{O}_{12}$; green tetrahedra represent $\mathrm{GeO}_{4}$ groups, yellow octahedra represent $L n / M \mathrm{O}_{6}$ groups and lilac circles represent $L n$ atoms on the $2 b$ site.

Figure 2 Temperature dependence of the molar magnetic susceptibility of $\mathrm{Y}_{2} \mathrm{CoGe}_{4} \mathrm{O}_{12}$. The inverse molar susceptibility is inset.

Figure 3 Observed and calculated neutron diffraction patterns; a difference curve is also shown. The upper and lower sets of reflection markers correspond to $\mathrm{CoGeO}_{3}$ and $\mathrm{Y}_{2} \mathrm{CoGe}_{4} \mathrm{O}_{12}$, respectively.

Figure 4 Normalised XANES data of a Co metal foil reference at 300K (green), $\mathrm{Y}_{2} \mathrm{CoGe}_{4} \mathrm{O}_{12}$ at $300 \mathrm{~K}$ (red) and $\mathrm{Y}_{2} \mathrm{CoGe}_{4} \mathrm{O}_{12}$ at $100 \mathrm{~K}$ (blue). The overlap between the latter two curves is nearly perfect and they are barely distinguishable.

Figure 5 Normalised EXAFS data of $\mathrm{Y}_{2} \mathrm{CoGe}_{4} \mathrm{O}_{12}$ at $300 \mathrm{~K}$ (experimental data in red, fit in black) and100 K (experimental data in blue, fit in black). The data in reciprocal space are shown on the left and those in real space on the right. The grey curve in reciprocal space shows the back-FT of the experimental within the window where the data have been fitted in real space, the $q$-space data (window in grey in the real-space plots). The plots in real space show both the magnitude of the FT data (full line) and also its real component (dashed line). 


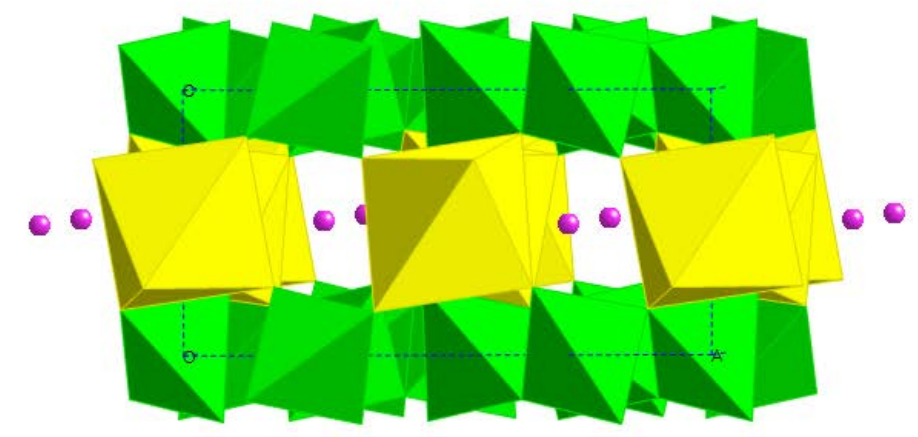

Figure 1

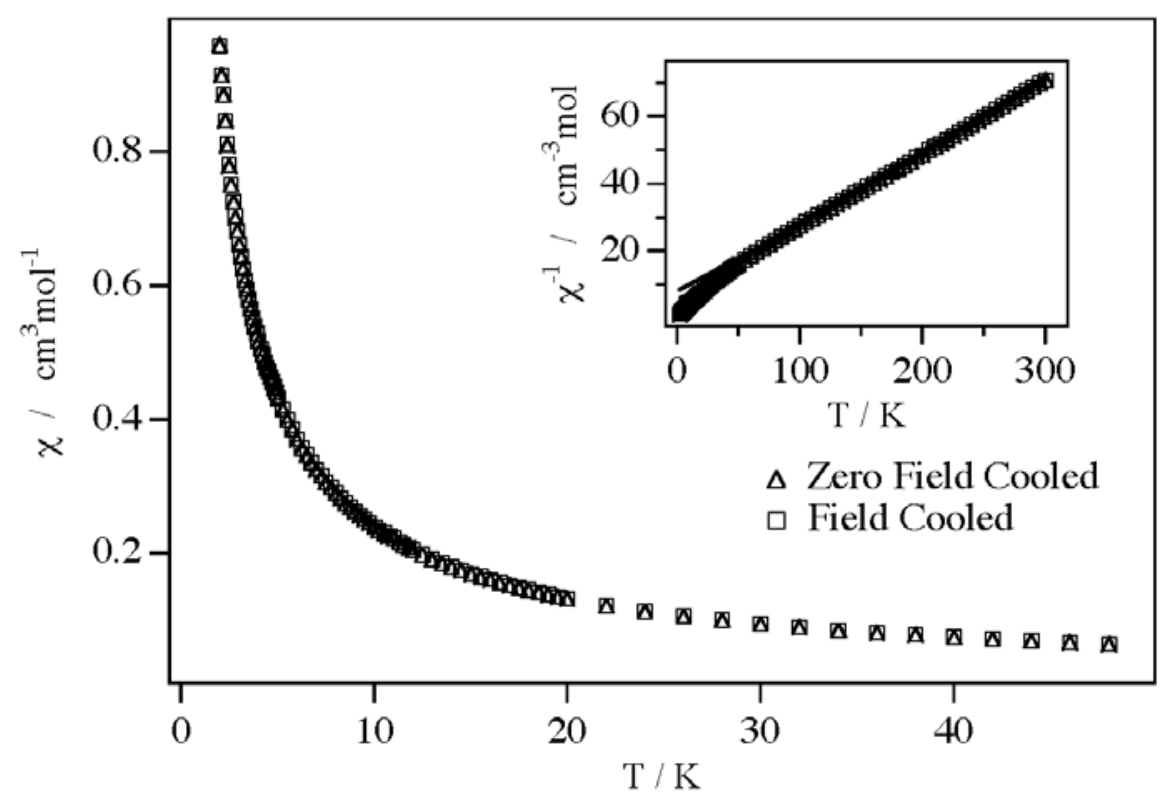

Figure 2 


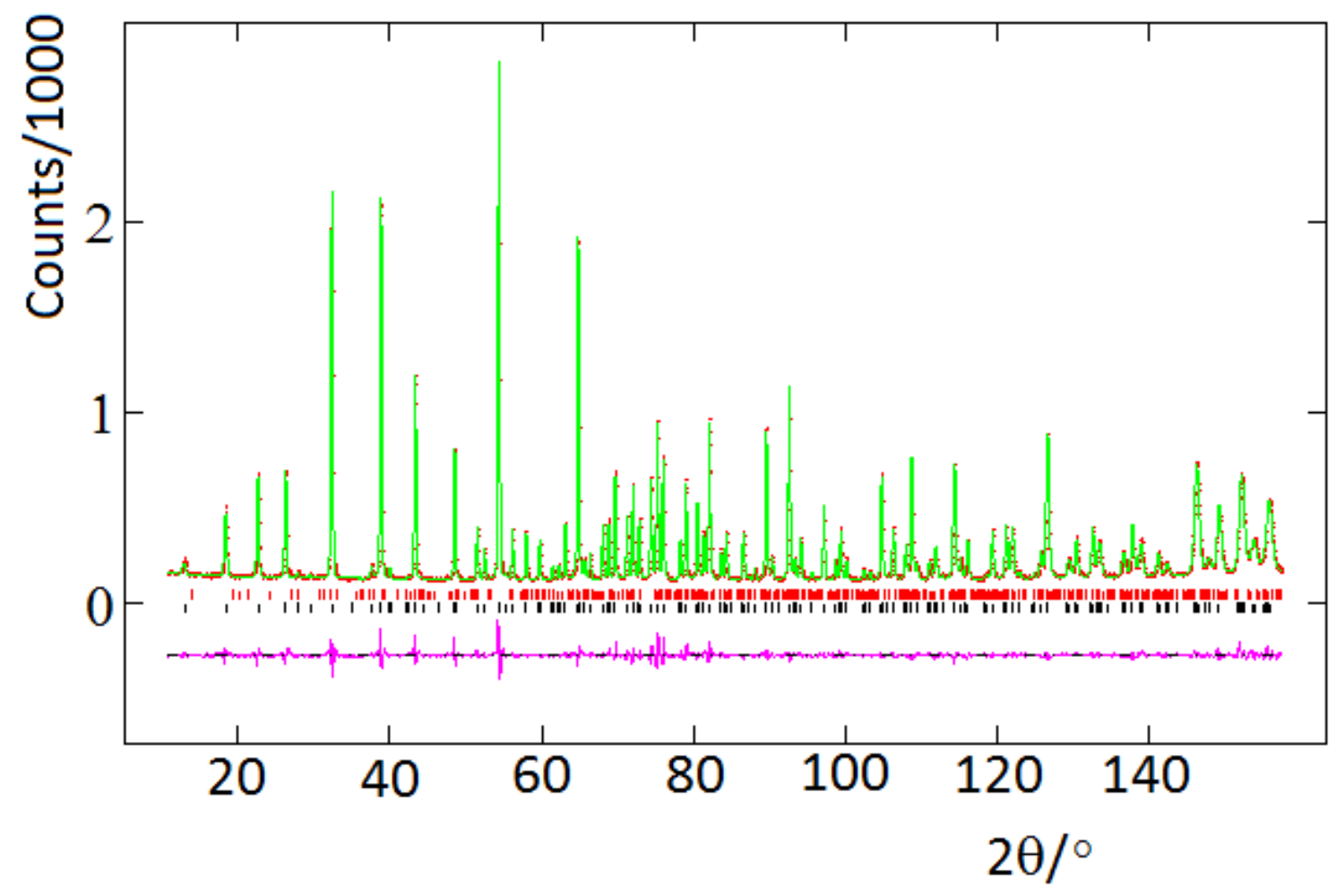

Figure 3

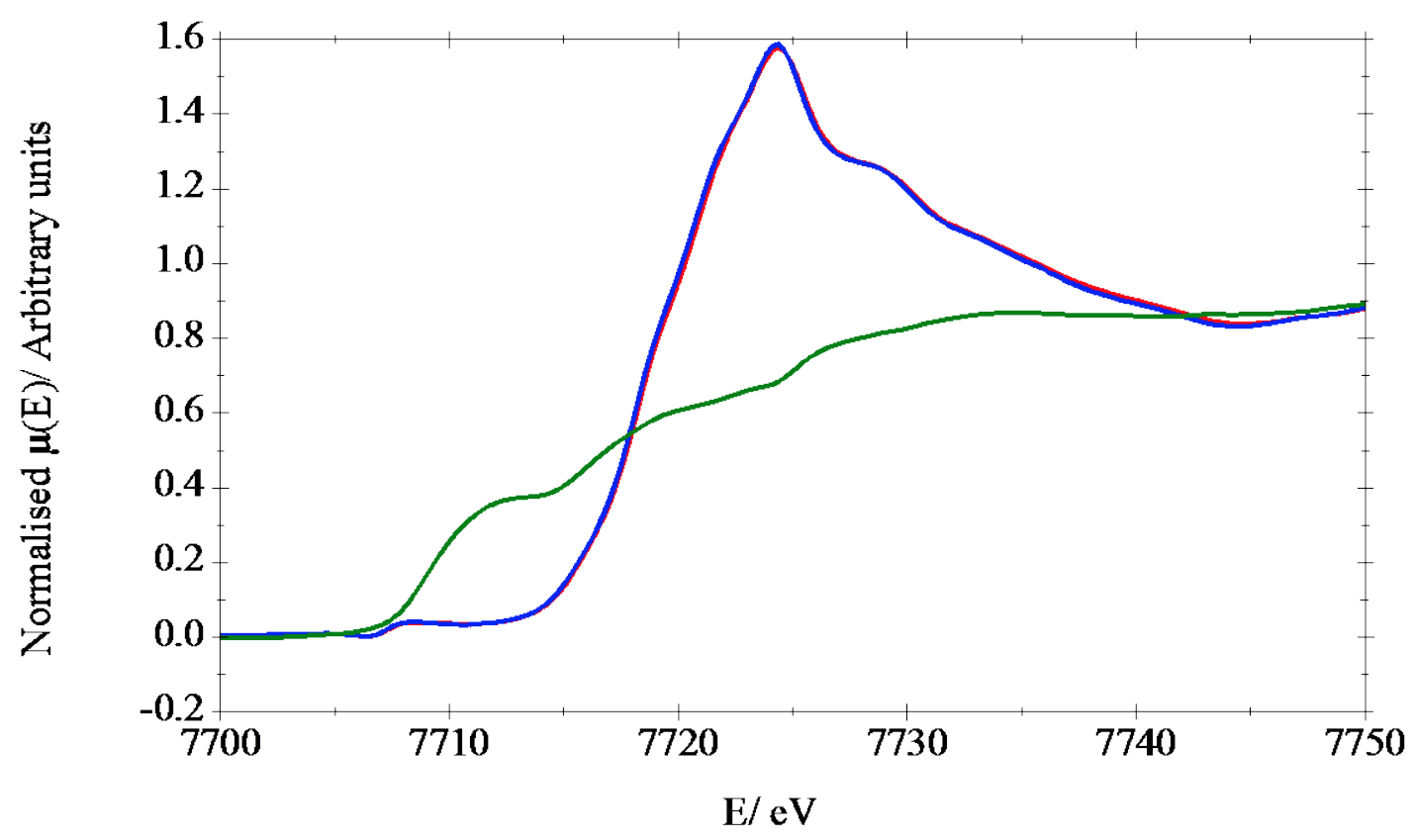

Figure 4 

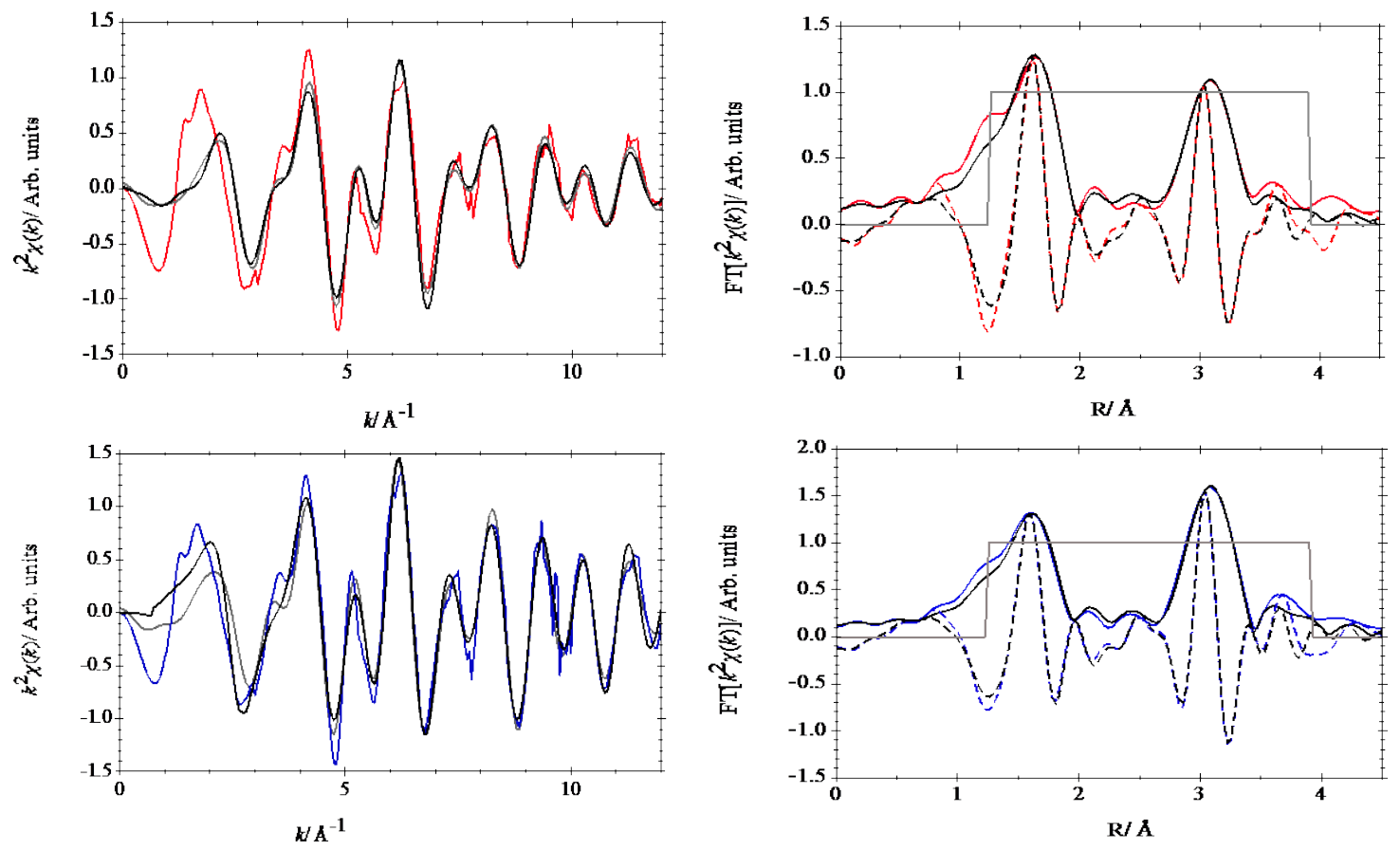

Figure 5 